\title{
Microbial reduction of graphene oxide by Azotobacter chroococcum
}

Youhu Chen, ${ }^{\mathrm{a}, \mathrm{b}}$ Yufang Niu, ${ }^{\mathrm{a}, \mathrm{b}}$ Tian Tian, ${ }^{\mathrm{a}}$ Juan Zhang, ${ }^{\mathrm{a}}$ Yuanfei Wang, ${ }^{\mathrm{a}, \mathrm{b}}$ Yunpeng Li, Lu-Chang Qin ${ }^{\mathrm{c}, *}$

${ }^{a}$ Ningbo Institute of Materials Technology and Engineering, Chinese Academy of Sciences, Ningbo, Zhejiang 315201, China

${ }^{b}$ University of Chinese Academy of Sciences, 19 A Yuquan Rd, Shijingshan District, Beijing, 100049, P.R.China

${ }^{c}$ Department of Physics and Astronomy, University of North Carolina at Chapel Hill, Chapel Hill, NC 27599-3255, USA

\begin{abstract}
We report an eco-friendly route for the reduction of graphene oxide (GO) by Azotobacter chroococcum at room temperature. Examinations using X-ray photoelectron spectroscopy, X-ray diffraction, and transmission electron microscopy indicate the formation of reduced graphene oxide ( $\mathrm{rGO}$ ) with a low degree of agglomeration. Quantitative analyses of electron diffraction data show that the produced rGO consists of single-layer sheets with a random stacking. It is suggested that the GO was reduced directly by the nitrogenase via sequential additions of electrons and protons followed by dehydration. This approach avoids the use of toxic chemical agents and also reduces the agglomeration of rGO.
\end{abstract}




\section{Introduction}

Graphene has attracted great attention in recent years due to its extraordinary chemical, electrochemical, mechanical, thermal, optical, and electrical properties. These excellent characteristics make it a promising material for potential applications in transistors, transparent electrodes, conductive inks, polymer composites, sensors and energy storage devices [1-4]. Many approaches have been developed for the synthesis of graphene to date, including micromechanical cleavage, liquid-phase or electrochemical exfoliation, epitaxial growth, chemical vapor deposition, reduction of dry ice, and chemical reduction of graphene oxide (GO) [5-9]. Among these methods, chemical reduction of $\mathrm{GO}$ is a most promising route towards a low cost and large-scale preparation of graphene or reduced graphene oxide (rGO) [9].

Certain chemical reducing agents are always used in the chemical reduction method. The common reducing agents include hydrazine [10], sodium borohydride (NaBH4) [11], and lithium aluminium hydride (LiAlH4) [12]. There are more than 50 types of reducing agents that have been reported in the recent years [9]. However, we are still facing a few issues in the preparation of graphene material of high quality. For example, the strong interactions between the stacked rGO sheets usually lead to irreversible agglomeration $[13,14]$. On the other hand, since any single reducing agent could not reduce all types of oxygen- containing functional groups associated with GO [13], combination of different reducing agents are often needed to scavenge the oxygen. In addition, the toxic nature of many chemical reducing agents makes them harmful to the environment.

In order to overcome the toxicity problem, many efforts have been attempted to explore green routes to achieve the reduction of GO. Especially, the usage of biological materials as reducing agents has received much attention in the recent years, which is more eco-friendly as compared with the conventional chemical agents. Ascorbic acid and L-cysteine are the typical examples in this regard as the reducing agents $[14,15]$. Phytoche-micals extracted from plants have also been utilized to reduce GO [16,17]. Salas et al. applied the respiration of Shewanella towards the reduction of graphene oxide [18]. 
In this work, we report an eco-friendly green approach for the reduction of GO using Azotobacter chroococcum at room temperature. Biological dinitrogen fixation is a key step in the global nitrogen cycle, where nitrogenase catalyzes the reduction of N2 to NH3. Nitrogenase has been demonstrated successfully to reduce other substances, such as acetylene [19]. Recently, nitrogenase has also been reported to catalyze the reduction of carbon monoxide $(\mathrm{CO})$ to produce methane, ethylene, ethane, propylene, propane, butylene, and butane [20,21]. Moreover, it was observed that the carbon dioxide $\left(\mathrm{CO}_{2}\right)$ could be reduced to $\mathrm{CO}$, formate, and methane by nitrogenase $[22,23]$. Furthermore, nitrogenase can reduce some carbon-containing compounds as well [24]. Since nitrogenase is always present in some Azotobacter, such as Azotobacter chroococcum, which has been widely used in agricultural production, biosynthesis, and microbial treatment $[25,26]$, it is therefore expected that the reduction of GO could be realized via Azotobacter chroococcum. In addition, the mild reactions should reduce agglomeration of $\mathrm{rGO}$ and the intermediate products generated in the nitrogen fixation process could also serve as the reducing agents to achieve N-doping.

\section{Experimental section}

\subsection{Preparation of graphene oxide}

Graphene oxide was obtained by oxidizing natural graphite powders according to a modified Hummers-Offeman method [25]. In a typical procedure, natural graphite powders $(3 \mathrm{~g})$ and $\mathrm{NaNO}_{3}(2 \mathrm{~g})$ were mixed in a beaker in an ice bath, followed by slow addition of $138 \mathrm{~mL} \mathrm{H}_{2} \mathrm{SO}_{4}$ with stirring. After $20 \mathrm{~min}, 18 \mathrm{~g}$ of $\mathrm{KMnO}_{4}$ was slowly added into the above mixture. The ice bath was then removed and the mixture was continuously stirred for 12 hours at room temperature. Subsequently, the mixture was diluted with $600 \mathrm{~mL}$ of deionized warm water $\left(60{ }^{\circ} \mathrm{C}\right) .20 \mathrm{~mL}$ of $\mathrm{H}_{2} \mathrm{O}_{2}(37 \%)$ was then added to this diluted solution for preventing further oxidation of the GO. The final mixture was washed with $\mathrm{HCl}(5 \%)$ and deionized (DI) water with centrifugation for 7 times, respectively. The precipitate was re-dispersed in $600 \mathrm{~mL}$ DI water and sonicated for 4 hours. After the solution was centrifuged at $11000 \mathrm{rpm}$ for $15 \mathrm{~min}$ to 
remove the precipitates, the GO powders were finally obtained with freeze-drying.

\subsection{Reduction of GO via Azotobacter chroococcum}

Azotobacter chroococcum was purchased commercially (Guangdong Microbiology Culture Center) and was cultured in solid medium at room temperature for 24 hours. Microbial reduction was initiated by mixing $100 \mathrm{mg}$ culture of Azotobacter chroococcum with $100 \mathrm{~mL}$ of as-prepared GO solution $(1 \mathrm{mg} / \mathrm{mL})$ with continuous stirring for 72 hours. Then the mixture was centrifuged to remove the residual bacteria. The supernatant was finally purified and freeze-dried to obtain the rGO powders.

\subsection{Characterization}

X-ray diffraction (XRD) was carried out at room temperature on a Bruker D8 Advance Davinici diffractometer with $\mathrm{Cu} \mathrm{K}_{\alpha 1}$ radiation. X-ray photoelectron spectroscopy (XPS) measurements were performed using a Shimadzu Axis Utltradld spectrometer with a monochromated Al Ka radiation $(h v=1486.6 \mathrm{eV})$. All XPS spectra were calibrated using the $\mathrm{C}$ 1s line at $284.6 \mathrm{eV}$. Curve fitting and background substraction were conducted by the usage of CASA XPS software. Transmission electron microscopy (TEM) images and electron diffraction (ED) patterns were acquired with Tecnai F20 operated at accelerating voltage of $200 \mathrm{kV}$ using powder samples that were suspended in alcohol by sonication and then transferred onto a lacey carbon coated grid.

\section{Results and Discussion}

Nitrogenase is an enzyme found in many bacteria and archaea that catalyzes biological dinitrogen fixation, the reduction of $\mathrm{N}_{2}$ to $\mathrm{NH}_{3}$ [24]. Three different classes of nitrogenase have been reported, among which Mo-nitrogenase is the most widely distributed and best-studied. Mo-nitrogenase contains two proteins designated as the Fe protein and MoFe protein, respectively. The Fe protein, with two ATP-binding sites, provides the energy for catalysis. The MoFe protein is the site of substrate binding and reduction. Catalysis is initiated when the Fe protein touches the MoFe protein, triggering intermolecular electron transfer [24]. The reduction process typically consists of the following step: (a) electrons are accumulated on the FeMo-cofactor of 
nitrogenase; (b) the substrates are bound on FeMo-cofactor; and (c) the reduction is finally achievable by the sequential additions of electrons and protons from nitrogenase to the bound substrates [28]. It has been demonstrated that cyclopropene could undergo reduction by Mo-nitrogenase to cleave the single bond forming propene [29]. It has also been shown that nitrogenase can reduce both $\mathrm{C}=\mathrm{S}$ and $\mathrm{C}=\mathrm{O}$ bonds, catalyzing the reduction of $\mathrm{CO}_{2}$ to $\mathrm{CH}_{4}[22,23]$. In addition, hydrazine is a product of dinitrogen reduction by vanadium-nitrogenase from Azotobacter chroococcum [30]. Given that the oxygen functionalities in GO contain hydroxyl, epoxide, carbonyl and carboxyl groups [9], it is suggested that the GO is reduced directly by nitrogenase as illustrated in Fig.1. The GO sheets were first bound on the MoFe protein of the nitrogenase. The $\mathrm{C}-\mathrm{O}-\mathrm{C}$ and $\mathrm{C}=\mathrm{O}$ bonds would then be cleaved to form $\mathrm{C}-\mathrm{O}-\mathrm{H}$ bonds by the additions of electrons and protons from nitrogenase, similar to the reduction of $\mathrm{CO}$ and $\mathrm{CO}_{2}$ [20-23]. The oxygen atoms were removed eventually by the reactions for dehydration reaction. In this pathway, due to the sequential additions of electrons and protons, the carbon at the graphene edges could turn to hydrocarbon, similar to the aromatic hydrocarbon. During the reaction, the $\mathrm{pH}$ value of the solution was indeed shifted in the acidic direction, which was also observed in the reduction of $\mathrm{C}_{2} \mathrm{H}_{2}$ [31].

The color change of the mixture after reaction, from brown to black, is a visual evidence to indicate the transformation of GO to graphene [9,32]. Fig. 2 shows the optical photographs of GO (before reaction) and rGO (after the reaction), which confirm the reduction of GO by Azotobacter chroococcum. The rGO was well dispersed in the aqueous solution due to the biological modification of GO. 
a

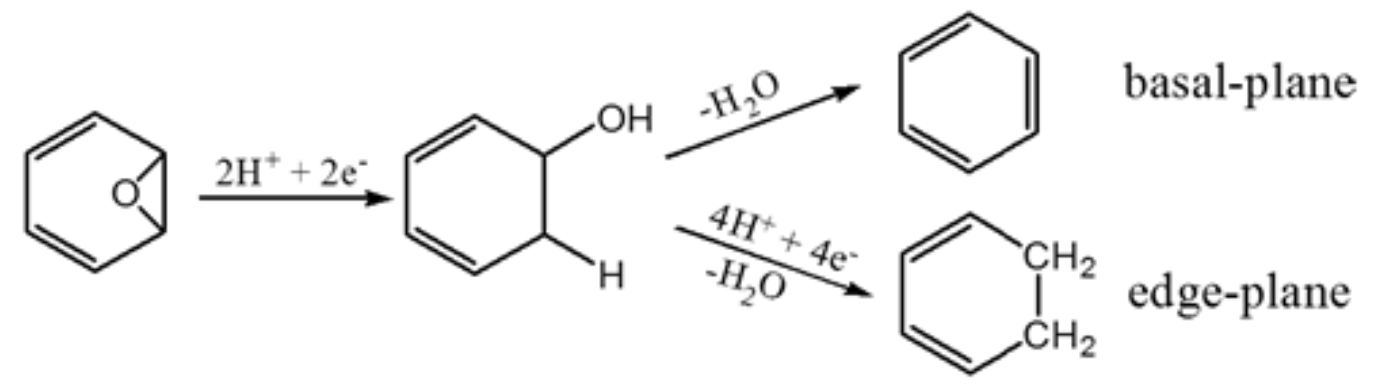

$\mathrm{b}$

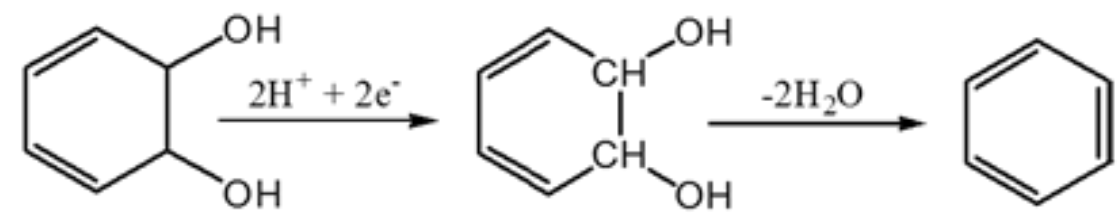

C

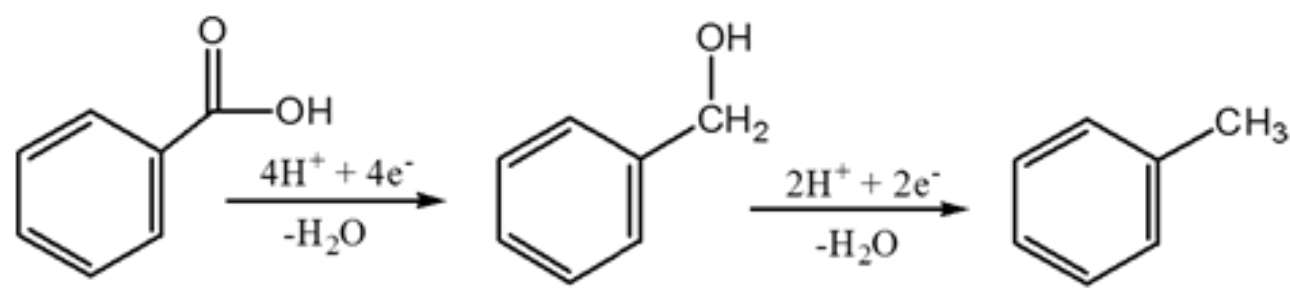

d

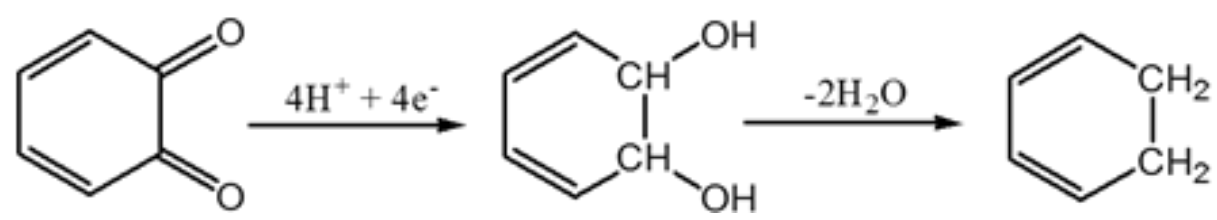

Fig. 1 Reduction of GO by nitrogenase with addition of hydrogen at (a) epoxy group on basal-plane or edge-plane, (b) hydroxyl group on basal-plane, (c) carboxyl group on edge-plane, and (d) carbonyl group on edge-plane.
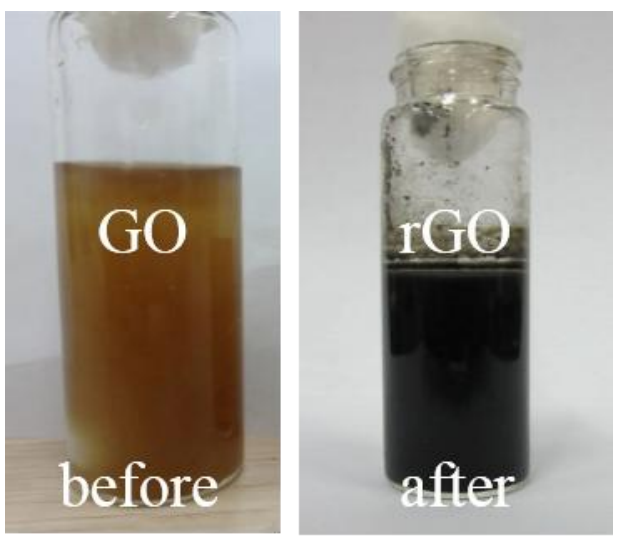

Fig. 2 Optical photographs of graphene oxide (left) and reduced graphene oxide with Azotobacter chroococcum (right) 
XPS was employed to assess the degree of GO reduction, using the atomic ratio of carbon and oxygen $(\mathrm{C} / \mathrm{O})$ acquired from the calculation of the ratio of respective area under the $\mathrm{C} 1 \mathrm{~s}$ and $\mathrm{O} 1 \mathrm{~s}$ peak. The $\mathrm{C} / \mathrm{O}$ ratio increased from 2.23 to 4.18 in our measured samples, indicating a successful reduction of GO. High-resolution C1s peaks in the XPS spectra were also used to reveal the functional groups on the GO and $\mathrm{rGO}$. Figure $3 \mathrm{a}$ shows that the $\mathrm{C} 1 \mathrm{~s}$ spectrum of the original $\mathrm{GO}$ contained two main components belonging to $\mathrm{C}=\mathrm{C} / \mathrm{C}-\mathrm{C}(\sim 284.6 \mathrm{eV})$ and $\mathrm{C}-\mathrm{O}$ (hydroxyl and epoxy groups, $\sim 286.6 \mathrm{eV}$ ), and one minor component ascribed to $\mathrm{C}=\mathrm{O}$ (carbonyl and carboxyl groups, $\sim 288.2 \mathrm{eV}$ ). Therefore, the oxygen-containing groups in GO consisted mainly of the epoxy and hydroxyl groups, which is in agreement with the Lerf-Klinowski model of GO [33]. On the other hand, the rGO (Fig. 3b) contained the same oxygen function-alities, but with greatly decreased intensities. However, the newly added C-N peak ( $285.7 \mathrm{eV})$ suggests formation of hydrazine groups during the reaction of GO with the released hydrazine, which would weaken the conjugate interactions between individual rGO sheets [10,34]. In addition, the F signal at 292 $\mathrm{eV}$ is attributed by contamination from the vacuum system [34]. These XPS data therefore show that the oxygen-containing functional groups in the original GO have been largely removed and a new nitrogen-containing functional group has been introduced with $\mathrm{N}$-doping in the final $\mathrm{rGO}$ product. 

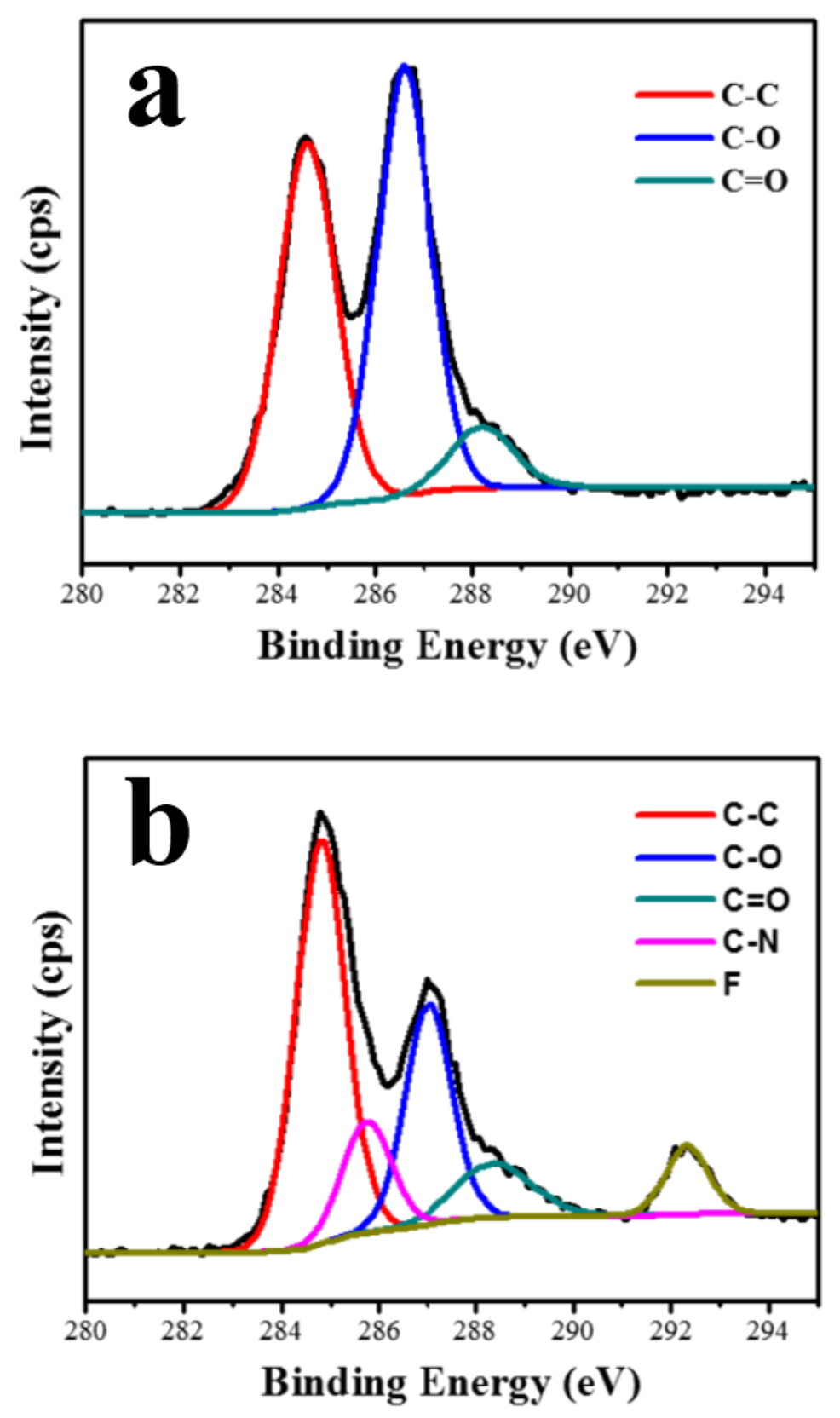

Fig. 3 The C1s peak in the XPS spectra of (a) GO and (b) rGO. C/O ratio has increased from 2.23 to 4.18 after reduction with simul-taneous $\mathrm{N}$-doping as a by-product.

The structure of rGO was further examined by XRD and TEM. The powder XRD patterns of natural graphite, GO, and rGO are shown in Fig. 4. The natural graphite exhibited a basal reflection (002) peak at $2 \theta=26.6^{\circ}\left(\mathrm{d}_{002}=0.335 \mathrm{~nm}\right)$. A typical broad peak near $11^{\circ}(\mathrm{d} \sim 0.81 \mathrm{~nm})$ was observed for the GO powders due to the 
formation of oxygen-containing groups between GO sheets. There is also a broad peak, with much weaker intensity, appeared in the range of $17 \sim 24^{\circ}$, corresponding d-spacing $0.37 \sim 0.52 \mathrm{~nm}$. After reduction by Azotobacter chroococcum, the peak at $11^{\circ}$ vanished and the broad weak peak in $17 \sim 24^{\circ}$ was retained, indicating parallel stacking of the rGO sheets. The disappearance of the reflection peak at $11^{\circ}$ is attributed to dismantle of regular stacking of the GO sheets in GO and therefore aggregation graphene is much reduced in $\mathrm{rGO}$.

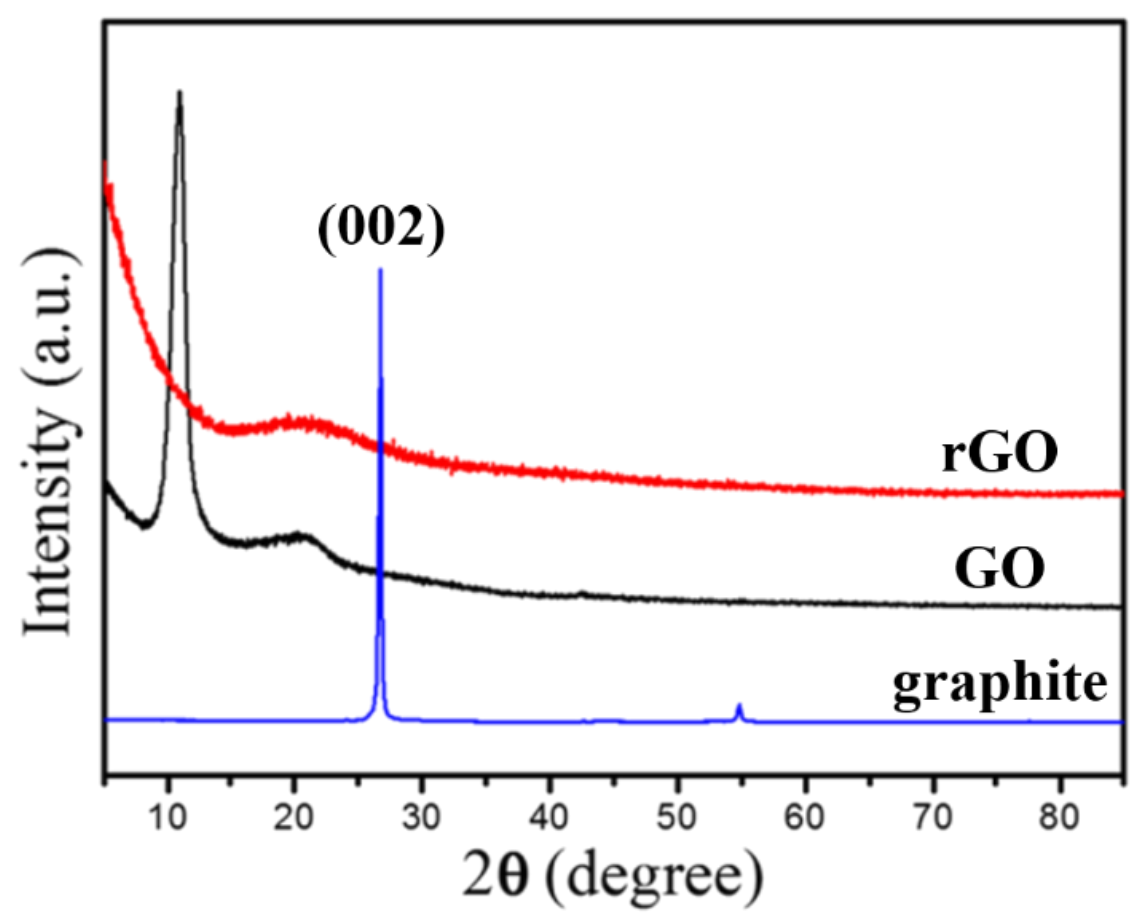

Fig. 4 X-ray diffraction patterns of natural graphite, GO and rGO. The characteristic graphitic peak $(\mathrm{d} 002=0.335 \mathrm{~nm})$ disappeared in GO and rGO. The typical GO peak $(\mathrm{d} \sim 0.8 \mathrm{~nm})$ is no longer visible in $\mathrm{rGO}$.

The individual structure of GO and rGO was studied by transmission electron microscopy (TEM). High-resolution transmission electron microscopy (HRTEM) images of folded edges can reveal the number of stacking layers [35,36]. While aberration-corrected HRTEM images could clearly determine the local chemical structure of GO and rGO at atomic resolution [37,38], electron diffraction is able to and often a more effective technique for identifying the layer number and stacking sequence $[35,36,39,40]$. In detail, for the Bernal stacking (ABAB...), the intensity 
ratio of $\mathrm{I}(100)$ to $\mathrm{I}(110)$ is larger than 1 for a monolayer graphene and it is less than 1 for multilayers stacks. For the AA stacking, the intensity ratio is the same and is greater than 1 for both monolayer and multilayer graphene [35]. The structure of GO has both oxidized areas with a random distribution of oxygen-containing functional groups and nonoxidized regions with only carbon atoms [33,38]. Fig. 5a shows a TEM image of GO sheet before reduction. The corresponding electron diffraction pattern (Fig. 5c) exhibited overlapped patterns indicating a turbostratic stacking of the GO sheets. Due to the disordered distribution of oxygen-containing functional groups, the diffraction spots are blurred, especially visible at the second ring corresponding to the (110) reflections. Fig. 5b shows a low-magnification TEM image of rGO. Selected-area electron diffraction (SAED) pattern (Fig. 5d) taken from the area labeled I showed a sharp hexagonal pattern. The measured intensity (Fig. 5g) confirmed that the intensity ratio of $\mathrm{I}(100)$ to $\mathrm{I}(110)$ is about 1.5 , indicating clearly that this area is monolayer graphene. Similar electron diffraction studies of area II (Fig. 5e and 5h) revealed that this region consisted of five single-layer rGO sheets stacked in a random manner after reduction. It should be pointed out that this single-layer rGO stacking is different from the strong irreversible stacking that is difficult to redisperse even with the ultrasound. The SAED pattern from area III (Fig. 5f) showed that the reflection spots were slightly blurred, similar to that of GO, indicating partial reduction of GO. Our explanation for these phenomena is that two factors important for the reduction of GO: (a) sufficient contact between GO and nitrogenase and (b) sufficient time for reduction reaction to occur and complete. Hence, satisfaction of both conditions resulted in complete reduction of individual sheets and partial reduction is attributed to the lack of either sufficient contact or sufficient time for reactions to complete. 

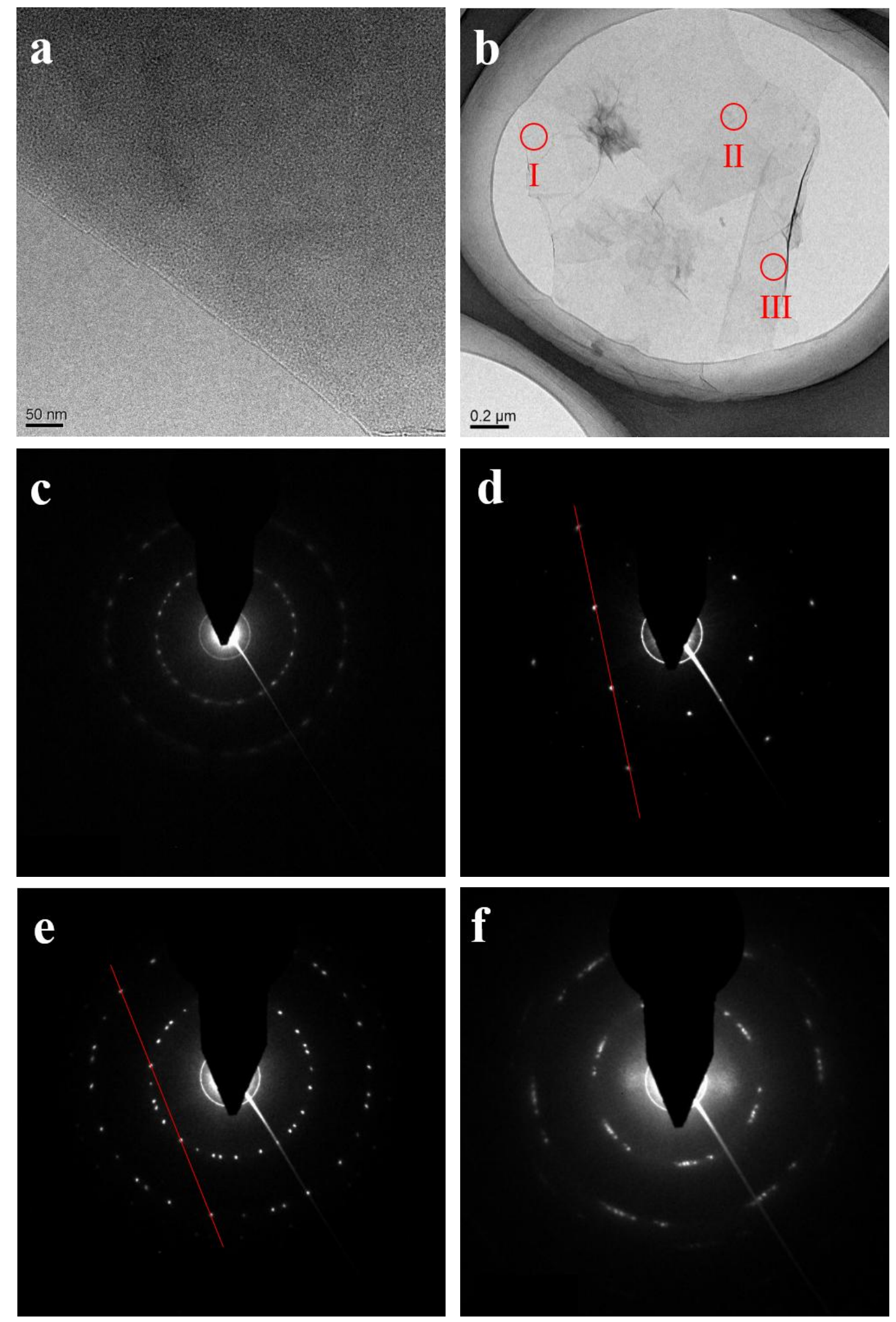

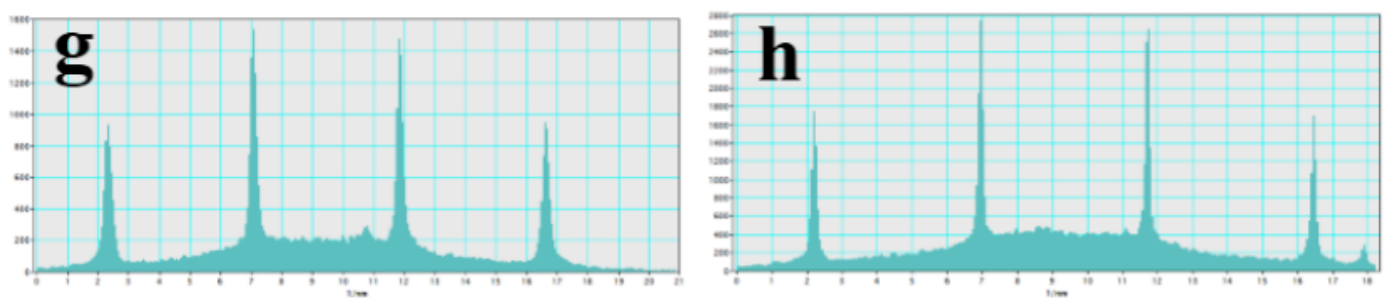

Fig. 5. Transmission electron microscopy images and electron diffraction patterns of GO and rGO. TEM images of (a) GO and (b) and rGO, respectively. (c) Selected-area electron diffraction patterns of GO; (d-f) SAED pattern from areas I, II, and III of rGO shown in (b). (g-h) Line scan of intensity measurement obtained from (d) and (e), respectively, showing both are from monolayer graphene.

\section{Conclusions}

An eco-friendly biochemical approach has been developed and applied to obtain graphene via reduction of graphene oxide by Azotobacter chroococcum at room temperature. The reduced graphene oxide is well dispersed in deionized water due to surface functionalization. While an average $\mathrm{C} / \mathrm{O}$ ratio of 4.18 was obtained from starting graphene oxide with $\mathrm{C} / \mathrm{O}=2.23$, complete reduction in individual sheets was identified with electron diffraction analysis. The pathway of reduction is that graphene oxide was reduced directly by the nitrogenase via the sequential additions of electrons and protons followed by the dehydration.

\section{Acknowledgements}

This study was partially supported by China National Science Foundation (Grant No. 11474294), Zhejiang Provincial Grant (No. LY14E020010), and Ningbo Innovative Team Grant (No. 2014B82004).

\section{References}

[1] K.S. Novoselov, A.K. Geim, S.V. Morozov, D. Jiang, Y. Zhang, S.V. Dubonos, I.V. Grigorieva, A.A. Firsov, Science 306 (2004) 666. 
[2] M.J. Allen, V.C. Tung, R.B. Kaner, Chem Rev 110 (2010) 132.

[3] K.S. Novoselov, V.I. Fal'ko, L. Colombo, P.R. Gellert, M.G. Schwab, K. Kim, Nature 490 (2012) 192.

[4] M.F. El-Kady, Y. Shao, R.B. Kaner, Nature Reviews Materials 1 (2016) 16033.

[5] Y. Zhang, L.Y. Zhang, C.W. Zhou, Accounts Chem Res 46 (2013) 2329.

[6] M. Zhou, J. Tang, Q. Cheng, G.J. Xu, P. Cui, L.-C. Qin, Chem Phys Lett 572 (2013) 61.

[7] J. Zhang, T. Tian, Y.H. Chen, Y.F. Niu, J. Tang, L.-C. Qin, Chem Phys Lett 591 (2014) 78 .

[8] M. Zhou, T. Tian, X.F. Li, X.D. Sun, J. Zhang, P. Cui, J. Tang, L.-C. Qin, Int J Electrochem Sc 9 (2014) 810.

[9] C.K. Chua, M. Pumera, Chem Soc Rev 43 (2014) 291.

[10] S. Stankovich, D.A. Dikin, R.D. Piner, K.A. Kohlhaas, A. Kleinhammes, Y. Jia, Y. Wu, S.T. Nguyen, R.S. Ruoff, Carbon 45 (2007) 1558.

[11] W. Gao, L.B. Alemany, L.J. Ci, P.M. Ajayan, Nat Chem 1 (2009) 403.

[12] A. Ambrosi, C.K. Chua, A. Bonanni, M. Pumera, Chem Mater 24 (2012) 2292.

[13] C.K. Chua, M. Pumera, Chem-Eur J 19 (2013) 2005.

[14] J. Gao, F. Liu, Y.L. Liu, N. Ma, Z.Q. Wang, X. Zhang, Chem Mater 22 (2010) 2213.

[15]D.Z. Chen, L.D. Li, L. Guo, Nanotechnology 22 (2011).

[16]Y. Wang, Z.X. Shi, J. Yin, Acs Appl Mater Inter 3 (2011) 1127.

[17]S. Thakur, N. Karak, Carbon 50 (2012) 5331.

[18]E.C. Salas, Z.Z. Sun, A. Luttge, J.M. Tour, Acs Nano 4 (2010) 4852.

[19]M.J. Dilworth, Biochim Biophys Acta 127 (1966) 285.

[20]C.C. Lee, Y.L. Hu, M.W. Ribbe, Science 329 (2010) 642.

[21]Y.L. Hu, C.C. Lee, M.W. Ribbe, Science 333 (2011) 753.

[22]L.C. Seefeldt, M.E. Rasche, S.A. Ensign, Biochemistry 34 (1995) 5382.

[23]Z.Y. Yang, V.R. Moure, D.R. Dean, L.C. Seefeldt, P Natl Acad Sci USA 109 (2012) 19644.

[24] L.C. Seefeldt, Z.Y. Yang, S. Duval, D.R. Dean, Biochimica Et Biophysica Acta 
-Bioenergetics 1827 (2013)1102.

[25] S. Viscardi, V. Ventorino, P. Duran, A. Maggio, S. De Pascale, M.L. Mora, O. Pepe, J Soil Sci Plant Nut 16 (2016) 848.

[26] G.A. Bonartseva, V.L. Myshkina, D.A. Nikolaeva, M.V. Kevbrina, A.Y. Kallistova, V.A. Gerasin, A.L. Iordanskii, A.N. Nozhevnikova, Appl Biochem Biotechnol 109 (2003) 285.

[27] W.S. Hummers, R.E. Offeman, J Am Chem Soc 80 (1958) 1339.

[28]B.M. Hoffman, D. Lukoyanov, Z.Y. Yang, D.R. Dean, L.C. Seefeldt, Chem Rev 114 (2014) 4041.

[29]C.E. Mckenna, M.C. Mckenna, M.T. Higa, J Am Chem Soc 98 (1976) 4657.

[30]M.J. Dilworth, R.R. Eady, Biochem J 277 (1991) 465.

[31]D.N. Pham, B.K. Burgess, Biochemistry 32 (1993) 13725.

[32]T. Kuila, S. Bose, P. Khanra, A.K. Mishra, N.H. Kim, J.H. Lee, Carbon 50 (2012) 914.

[33]A. Lerf, H.Y. He, M. Forster, J. Klinowski, J Phys Chem B 102 (1998) 4477.

[34]H.A. Becerril, J. Mao, Z. Liu, R.M. Stoltenberg, Z. Bao, Y. Chen, Acs Nano 2 (2008) 463.

[35]S. Horiuchi, T. Gotou, M. Fuijwara, R. Sotoaka, M. Hirata, K. Kimoto, T. Asaka, T. Yokosawa, Y. Matsui, K. Watanabe, M. Sekita, Jpn J Appl Phys 242 (2003) L1073.

[36]J.C. Meyer, A.K. Geim, M.I. Katsnelson, K.S. Novoselov, T.J. Booth, S. Roth, Nature 446 (2007) 60.

[37]K. Erickson, R. Erni, Z. Lee, N. Alem, W. Gannett, A. Zettl, Adv Mater 22 (2010) 4467.

[38]C. Gomez-Navarro, J.C. Meyer, R.S. Sundaram, A. Chuvilin, S. Kurasch, M. Burghard, K. Kern, U. Kaiser, Nano Lett 10 (2010) 1144.

[39]J.C. Meyer, A.K. Geim, M.I. Katsnelson, K.S. Novoselov, D. Obergfell, S. Roth, C. Girit, A. Zettl, Solid State Commun 143 (2007) 101.

[40]Y. Hernandez, V. Nicolosi, M. Lotya, F.M. Blighe, Z.Y. Sun, S. De, I.T. McGovern, B. Holland, M. Byrne, Y.K. Gun'ko, J.J. Boland, P. Niraj, G. Duesberg, 
S. Krishnamurthy, R. Goodhue, J. Hutchison, V. Scardaci, A.C. Ferrari, J.N. Coleman, Nat Nanotechnol 3 (2008) 563. 


\section{Graphical Abstract}
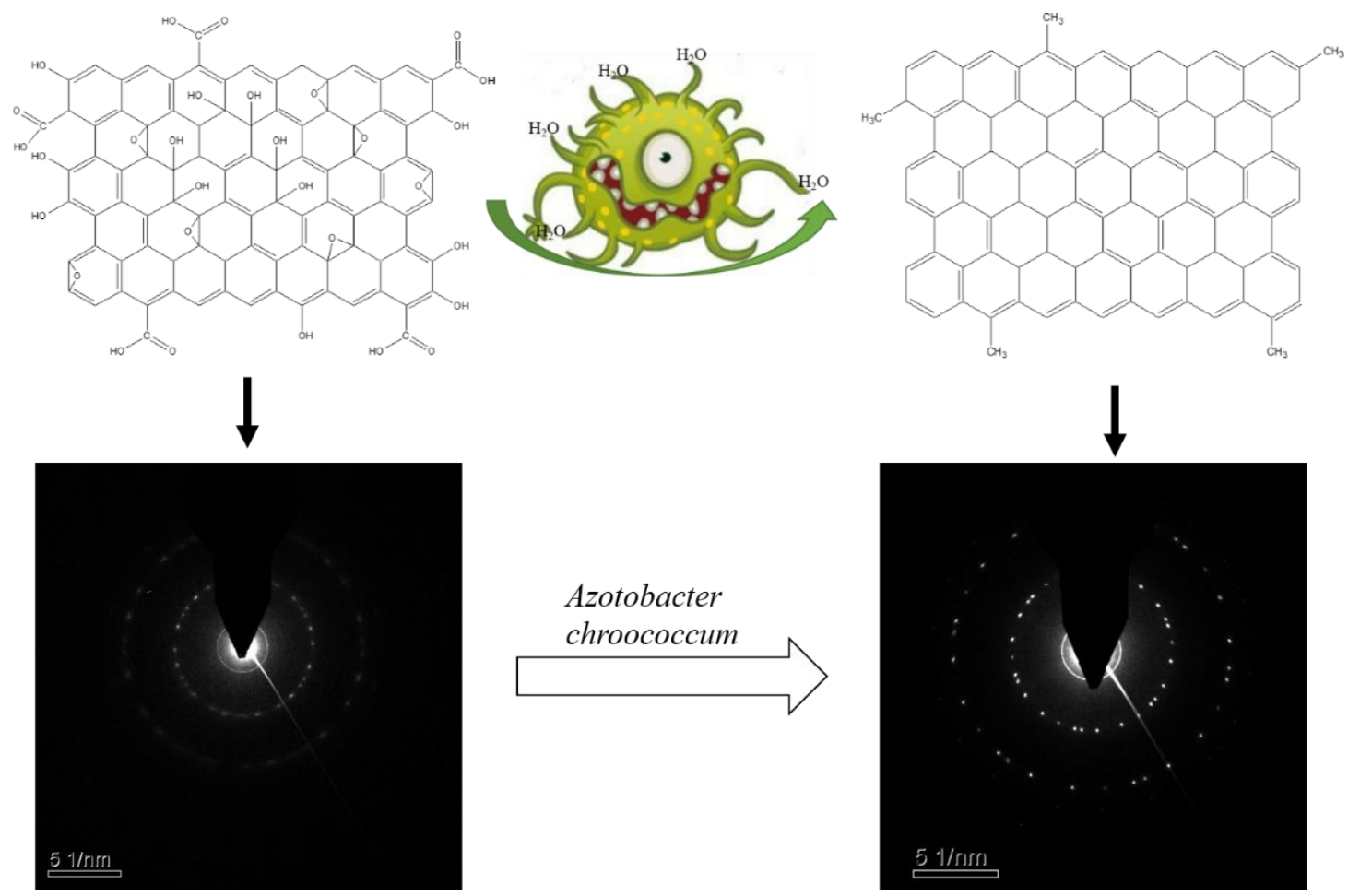